\title{
Parametric Study on Failure Mode of the Bearing Support under FBO Load
}

\author{
Zhimei Zhao a , Huiying Song and Zhennan Tang \\ AECC Commercial Aircraft Engine, Shanghai 201108, China \\ ahelenzhao5@hotmail.com
}

Keywords: Aero-engine, FBO, primary fuse, Finite element method.

\begin{abstract}
A typical high bypass ratio turbofan engine is used in this paper to study the structural parameters for the fuse, and a hollow thin section on the support of bearing No.1 between a forward bearing seat and an aft mount flange is referred to as the primary fuse structure. Influences of its structural parameters on failure mode of the bearing support under FBO load are discussed based on the explicit dynamic finite element method. As the result of parametric study, the following conclusions are obtained. Shear buckling failure of the bearing support is easy to occur under FBO load when the thin section is near to the front flange. And axial buckling failure of the bearing support is easy to occur when the thin section is near to the rear flange. Thickness of the thin section is directly related to the FBO peak load at the No.1 bearing. Plastic deformation in the form of wrinkling circumferentially around the thin section is observed when the axial width of the thin section is substantially greater than its wall thickness.
\end{abstract}

\section{Introduction}

During its operation, especially during take-off and landing, aircraft might be hit by foreign objects such as birds. Compared to other components of an airplane, engine has the highest proportion of been hit (43.13\%) [1]. Birds impacting on engine may lead one piece or more pieces of fan blade broken and separated from the remainder of the fan, namely FBO (Fan Blade Off). For high-speed rotating engines, the FBO event will have a huge impact and unbalanced load [2], which may cause catastrophic accidents such as failure of the engine mounting system, uncontrollable fire, and engine lost thrust. In response to the serious threat posed by the FBO incident, national aviation administrations have made regulations in the Airworthiness Regulations [3-5] to ensure that the aircraft is still safe to operate after the FBO incident.

FBO event itself is hard to avoid so that effective measures must be taken in the engine design stage to protect the engine under the FBO load. In order to improve capacity of the engine to survive under FBO load without increasing its weight significantly, the concept of fuse design [6] is introduced into engine design. The so-called fuse design is referred to the introduction of a mechanically weakened section to some of the components of the engine as sacrificial units (fusing parts), which fail under the FBO load. As a result, this would change the dynamic characteristics and the load path of the engine, ultimately reducing the load transmitted to the critical components of the engine [7], such as mounts.

Once a fan blade is released, the low-pressure (LP) rotor is subjected to an impulsive force at the fan-disk acting radically outward due to the introduction of sudden imbalance. And a substantial load created in the damaged fan would transmit to the fan frame, mounting systems mainly through bearing No.1, bearing No.2 and their supports. Thus fuse structures are often placed near them. At present, major aviation engine companies have applied a series of patents about fuse design [8-10]. They are mainly involved in a hollow thin section on the support of bearing No.1 as primary fuse and shearing pins set between the inner and outer ring of bearing No.2 as secondary fuse. If FBO event occurs, both the thin section on the support of bearing No. 1 and shearing pins at bearing No. 2 would fail. The failure of the thin section releases the constraint of the fan shaft at bearing No.1, and the failure of the shearing pins release the radial and pitch rotation constraint of the fan shaft at bearing No.2. As a result, the critical speed of the low-pressure rotor is reduced, changing from the subcritical state to 
the supercritical state. The load path from the damaged fan to the static frames is changed. As a result, loads transmitted to the critical components such as fan frame and mounting systems are significantly reduced.

The hollow thin section is a common type of fuse design in engineering [8], its structural parameters will directly affect the failure mode of the support of bearing No.1 under FBO load, and then influence the load reduction effect. Although researchers have carried out relevant analyses on FBO events [11-14] and dynamic characteristics of the bearing support, the influences of structural parameters of the thin section are rarely reported. In this paper, the thin section is set as primary fuse, and influences of its structural parameters on the failure mode of the support are analyzed based on a full-engine model with both implicit buckling analysis and explicit dynamic analysis. The relevant conclusions are useful for the design of primary fuse structure.

\section{Finite element model}

The threshold for the failure of the thin section should be identified at first during fuse design, which is determined by FBO load of the engine. As the full-engine model is very complex and FBO event is a highly nonlinear transient impact process, the nonlinear finite element software LS-DYNA is used for explicit dynamic calculations to obtain the FBO load. The model is relatively simplified by not considering some irrelevant details, such as squeezed film dampers, friction in the bearings, air and temperature effects, etc.

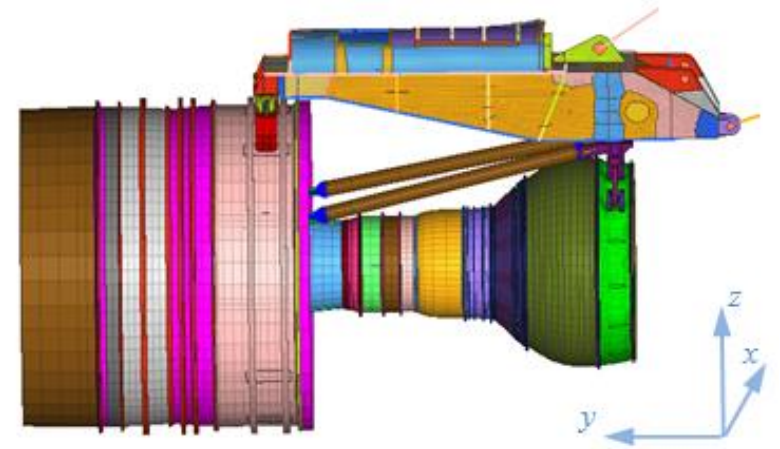

Fig.1 Full-engine element model

The full-engine 3D finite element model is shown in Fig. 1, where the $X$ axis is in the horizontal direction, the $\mathrm{Y}$ axis is in the axial direction, and the $\mathrm{Z}$ axis is in the vertical direction of the engine. The finite element model is mainly made of eight-noded reduced integration constant stress brick elements, while shell elements are used for blades and as coating elements between blades and disks to minimize the hinge effect. Tied contacts are used to join both flanges of stationary cases and flanges of the rotors. The mounting system is constrained to simulate the boundary conditions of the engine attached to the aircraft wing structure.

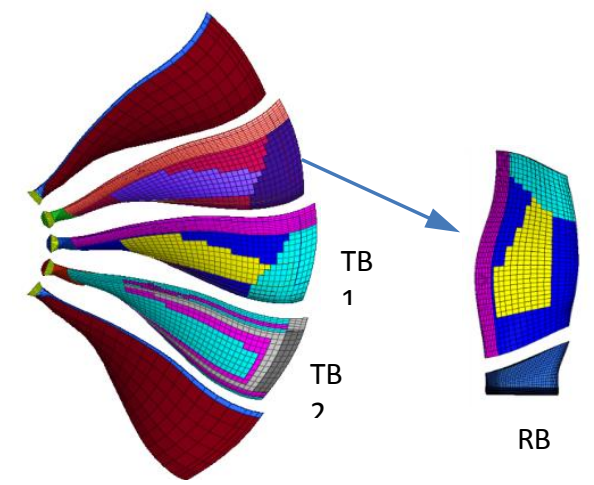

Fig.2 Finite Element Model of the Fan Blades

The FBO model consists a fully-bladed fan structure, including three fine- meshed blades and all other relatively coarse-meshed blades. All fan blades and fan casing are made of composite material. 
At the beginning of the calculation, the releasing blade is released from the flow path to simulate the FBO event, as shown in Fig 2.

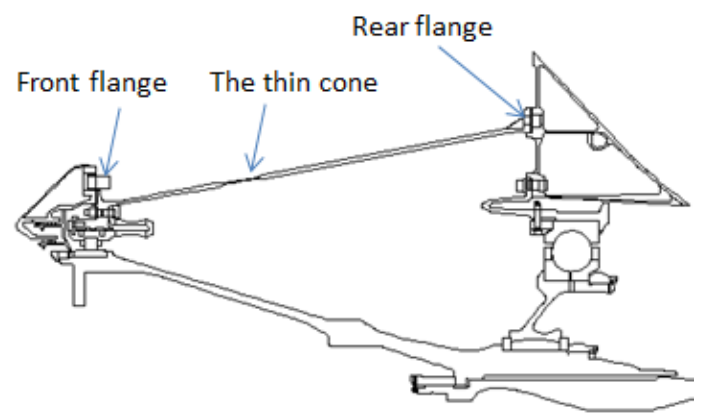

Fig.3 The support of bearing No.1

The front flange of the support of bearing No.1 is connected to its seat, while the rear flange is connected to the inner flange of the fan frame case, as shown in Fig. 3. A hollow thin section is placed on the support of bearing No.1between the front flange and the rear flange as the primary fuse.

\section{Mechanical Behavior of the Bearing Support without Fuse Design}

The load characteristics of the bearing support are analyzed, including the external input loads at both flanges of the support and the internal stress distribution, which are useful for the influence analysis of the structural parameters on the support failure mode.

\subsection{The loads at both flanges of the bearing support}

The bearing support without fuse design has enough strength to avoid being damaged during normal operation, thus the loads at the front and rear flanges are nearly equal in amplitude and opposite in direction. Normalized FBO loads at the front flange of the bearing support are shown in Fig 4. The curves show that loads in the $\mathrm{X}$ and $\mathrm{Z}$ directions at the front flange fluctuate over time, and the fluctuation period is proportional to the fan rotation speed. The amplitude of the radial load at the flange increases rapidly after the FBO event, then maintains a high level and fluctuates slightly. The sudden increase of loads at the flanges of the bearing support after RB released is utilized for further fuse design. And the thin section is designed to fail during this load increasing process.

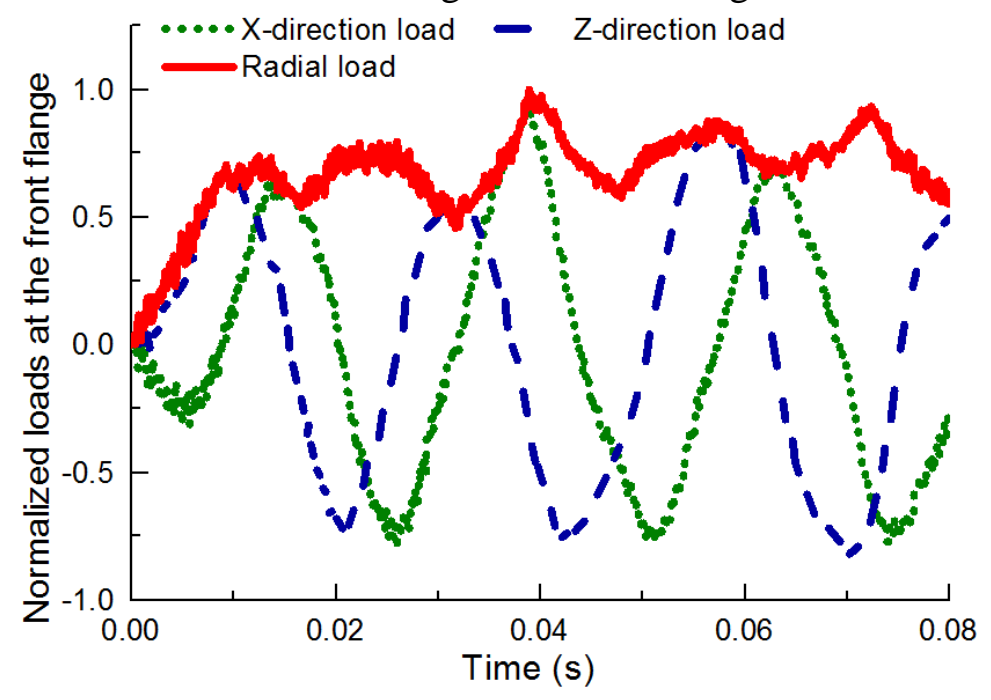

\subsection{The internal stress of the bearing support}

Fig.4 The loads at the front flange of the bearing support

Once FBO event occurs, loads at the front and rear flanges of the support of bearing No.1 fluctuate over time, as shown in Figure 4. The radial load $\mathrm{F}$ at the front flange of the bearing support is in opposite direction of the falling-off mass at the moment $\mathrm{RB}$ is released. The radial load $\mathrm{F}$ will rotate with the fan, transmitting to the rear flange and the fan frame. Due to constraints at the flanges, the radial load $\mathrm{F}$ results in a reaction force with equal amplitude but opposite direction at the rear flange of the bearing support, forming a pair of couple, as shown in the left figure of Fig 5. This pair of 
couple will cause the bending moment in the cross-section of the support, making one side of the support pulled and the other side pressed.
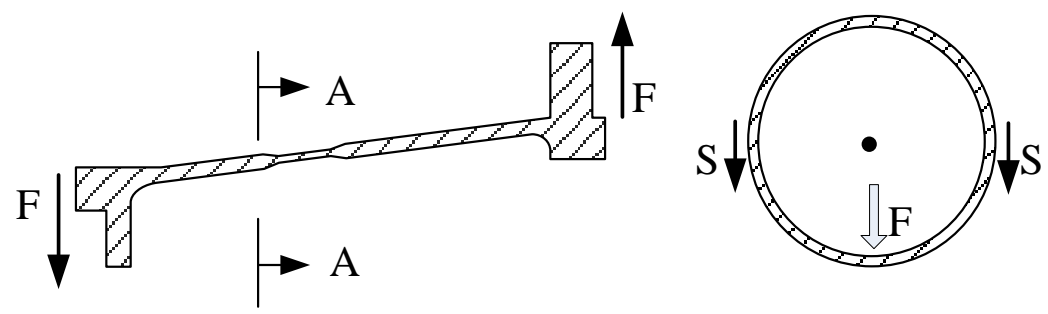

Fig.5 Scheme of load state for the bearing support

In addition, the rotated and unbalanced load $\mathrm{F}$ also produces a shear force $\mathrm{S}$ in the cross section of the support, resulting in a corresponding shear stress. As shown in the right figure in Fig. 5, the maximum shear stress appears at a position $90^{\circ}$ apart from the unbalanced load $\mathrm{F}$ in the circumferential direction. When the unbalanced load $\mathrm{F}$ is at the 6 o'clock position, the maximum shear stress occurs at the 3 o'clock and 9 o'clock positions in the circumferential direction of the support, respectively.
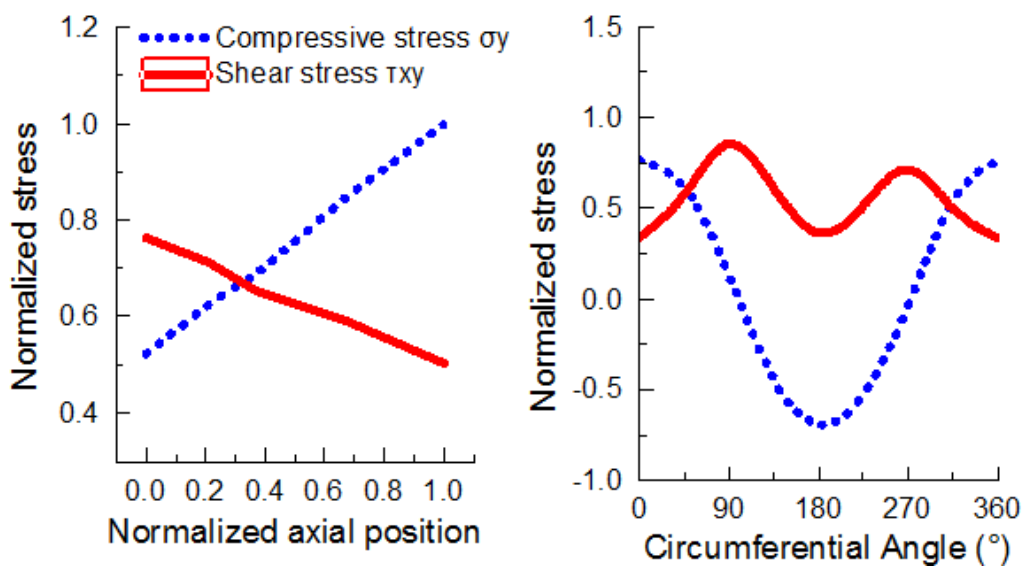

Fig.6 Distribution of the stress at axial and circumferential directions

Distribution of the maximum compressive stress and shear stress on the bearing support along the axial and circumferential directions are shown in Fig 6. The left graph shows that the compressive stress increases from the front flange to the rear flange, while the shear stress exhibits an opposite trend. This is because the bending moment becomes greater when it is getting closer to the rear flange. Curves in Fig. 6 represent the relationship between the shear stress and the compressive stress along the circumference in a cross section near the front flange of the bearing support. The absolute value of the maximum shear stress $\tau_{\mathrm{xy}}$ is greater than that of the compressive stress $\sigma_{\mathrm{y}}$.

\section{Influences of fuse structural parameters}

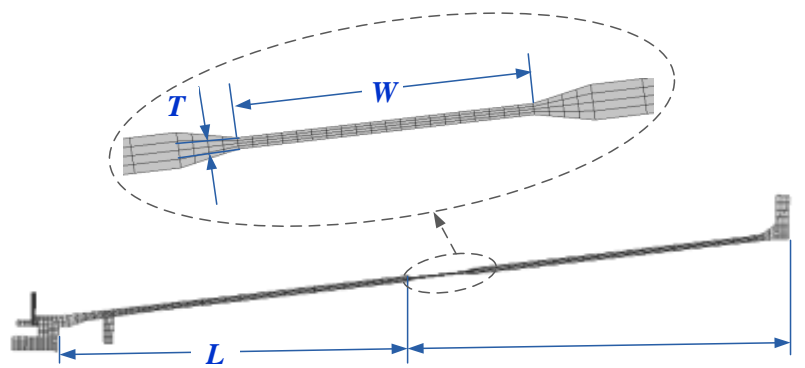

Fig.7 Finite Element Model for the Support of Bearing No.1

The structure of the bearing support with fuse design is shown in Figure 7, and a hollow thin section is referred as primary fuse structure. For simplification, the thin section is symmetrically distributed along the centerline of the non-thinning parts. Influence parameters are defined as its axial position $\mathrm{L}$, wall thickness $\mathrm{T}$ and axial width $\mathrm{W}$, as shown in Fig. 7. The failure mode of the bearing 
support will be analyzed based on the implicit buckling mode calculations and explicit dynamic method.

\subsection{The axial position of the thin section}

The axial position $\mathrm{L}$ of the thin section should be determined at first during fuse design. A submodel of the bearing support without fuse design is established for implicit buckling mode calculation in ANSYS, and its rear flange is fixed. The radial force F is loaded on the contact surface at the front flange of the bearing support through RBE3, as shown in Fig 8.

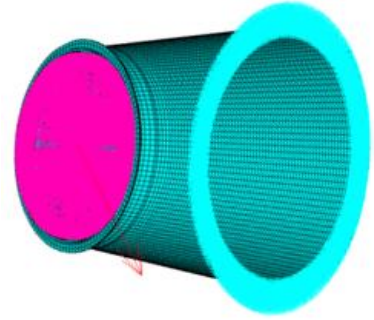

Fig.8 ANSYS model of the bearing support

The buckling modal analysis is performed for the bearing support in as shown in Fig. 8. The first order of the buckling mode is shown in Fig. 9. For the thin-wall structure of the bearing support, which is constrained at one flange and applied the radial force at the other, the first buckling mode is circumferential buckling. In the circumferential direction, the maximum buckling positions are about $50^{\circ}$ with respect to the plane defined by the central axis and the radial force $\mathrm{F}$ symmetrically. This indicates that the circumferential buckling is caused as a result of combination of the compressive stress and the shear stress, and the shear stress plays a dominant role. The total length of the bearing support is $\mathrm{L}_{\text {total }}$, distance from the maximum deformation position of the first buckling mode to the front flange is $\mathrm{L}_{0}$. For the current case, the maximum deformation position of the first buckling mode is $\mathrm{L}_{0}=\frac{8}{25} \mathrm{~L}_{\text {total }}$.
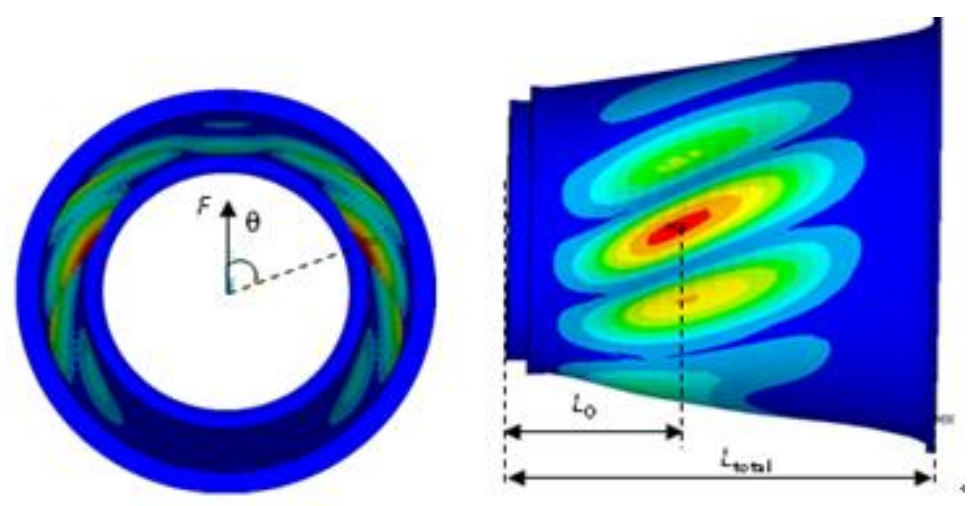

Fig.9 Buckling mode of the support without fuse design

A hollow thin section is placed on the bearing support. Its thickness is $50 \%$ of the thickness of the non-thinning section, and ratio of its axial width and wall thickness is 30 . The axial position of the thin section on the bearing support isL. A case study is carried out for different positions of this section. Cases for $\mathrm{L}=4 / 25 \mathrm{~L}_{\text {total }}, \mathrm{L}=8 / 25 \mathrm{~L}_{\text {total }}, \mathrm{L}=12 / 25 \mathrm{~L}_{\text {total }}$ and $\mathrm{L}=16 / 25 \mathrm{~L}_{\text {total }}$ are calculated separately, and contour diagrams for their first buckling modes are obtained, as shown in Fig 10.

Fig. 10 shows that the maximum deformation positions of the first buckling mode are getting closer to the thinning section after application of the thin section on the bearing support. Buckling coefficients corresponding to figure 10 are shown in Table 1 . The buckling coefficient of the bearing support increases firstly, and then decreases with the axial position of the thin section moved from the front flange to the rear flange. There is an axial position that the buckling coefficient of the bearing support is larger than that of other cases, and the failure is less likely to occur. For the current case, the buckling coefficient of the bearing support is largest when the axial position of the thin section is $L=8 / 25 L_{\text {total }}$. At this situation, the thin section is located near the maximum deformation position of the first buckling mode for the support without fuse design. 


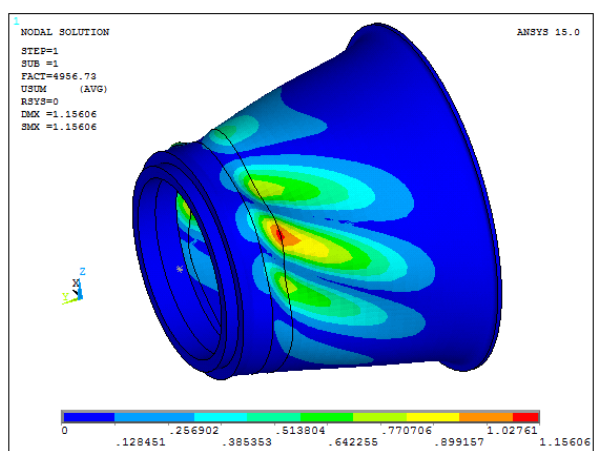

(a) $L=4 / 25 L_{\text {total }}$

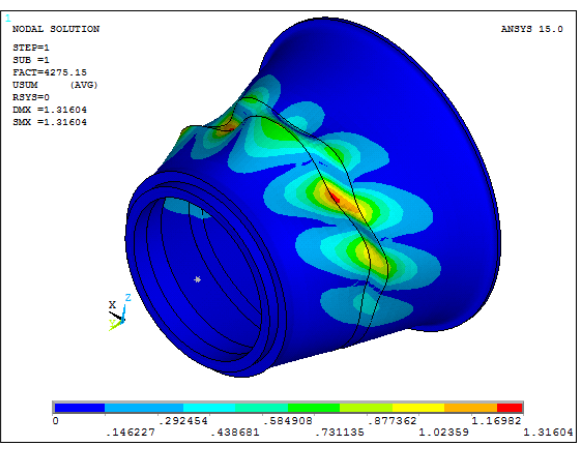

(c) $L=12 / 25 L_{\text {total }}$

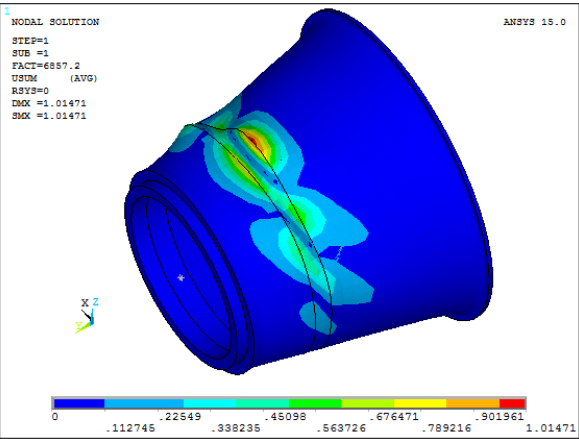

(b) $L=8 / 25 L_{\text {total }}$

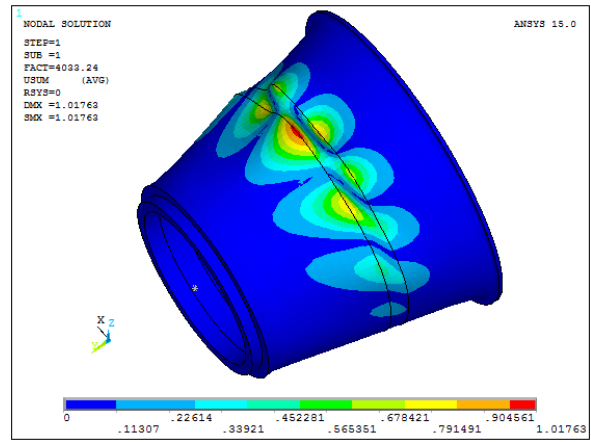

(d) $L=16 / 25 L_{\text {total }}$

Fig.10 Buckling mode of the support with a thin section at different axis position

The buckling coefficient of the bearing support changes with the axial position of the thin section because the shear stress decreases gradually with the axial position and the compressive stress increases with the axial position, as shown in Fig 6. When the thin section is close to the front flange, the circumferential shear buckling failure is likely to occur. When it is close to the rear flange, the axial buckling failure is liable to occur. In the design process, it is desirable that the primary fusing part does not undergo axial buckling failure under any working condition, but the circumferential shear buckling failure occurs under the FBO condition, so the axial position of the thin section should not be far away from the front flange.

Table 1 .The buckling coefficient for cases of thin section at different axial positions

\begin{tabular}{|c|c|c|c|c|}
\hline $\begin{array}{c}\text { Axial position of the thin section } \\
\boldsymbol{L} / \boldsymbol{L}_{\text {total }}\end{array}$ & $\mathbf{4 / 2 5}$ & $\mathbf{8 / 2 5}$ & $\mathbf{1 2 / 2 5}$ & $\mathbf{1 6 / 2 5}$ \\
\hline Buckling coefficient & 4957 & 6857 & 4275 & 4033 \\
\hline
\end{tabular}

\subsection{The thickness of the thin section}

As the primary fuse structure, the thin section is thinner than other area, and as such would fail in circumferential shear buckling under FBO load. Therefore, thickness of the thin section is closely related to the amplitude of the FBO load. As indicated in Fig. 4, it is determined by the FBO peak load suffered by the support of bearing No.1.

The thin section is designed to locate between the front flange of the support and the maximum deformation position of the first buckling mode. Influence of its thin section wall thickness $\mathrm{T}$ is discussed below. The ratio of its axial width and wall thickness is 30 . Initial value of $\mathrm{T}$ is $95 \%$ of the thickness of the non-thinning section. Explicit dynamic calculations are carried out. If the thin section does not fail under current thickness, the wall thickness $\mathrm{T}$ is reduced by $5 \%$ each run. The explicit dynamic calculations are performed again until the thin section fails quickly under the FBO load. 

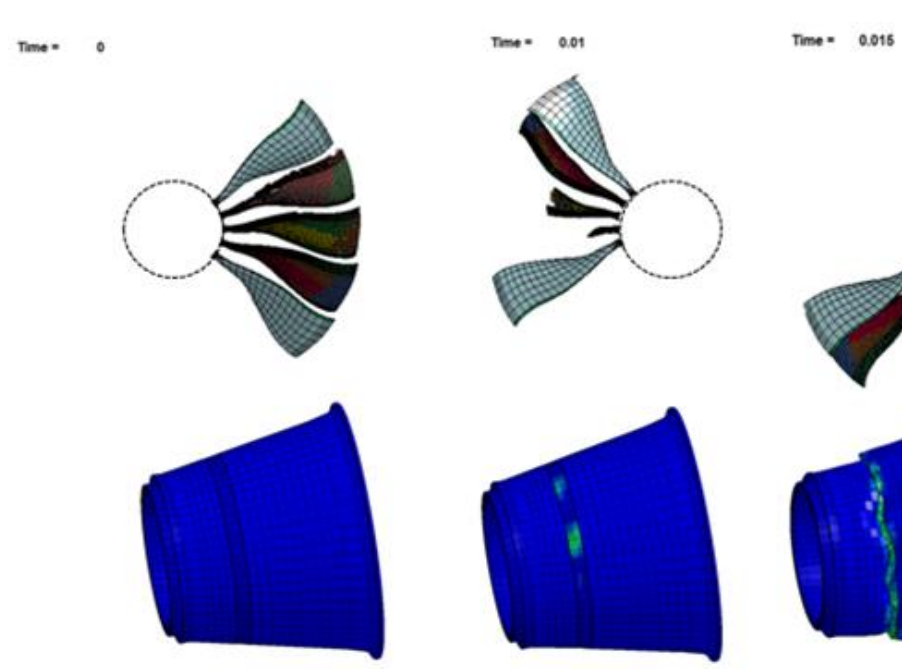

Fig.11 Effective plastic strain of bearing No.1 at different time

For the current example, when the wall thickness $\mathrm{T}$ is $60 \%$ of the non-thinning section, the thin section will fail rapidly after the release of RB. It is considered that the current thickness of the thin section is feasible. Effective plastic strains of the bearing support at different times are shown in Figure 11. It can be seen that the plastic strain has appeared about $0.5 \mathrm{~ms}$ after the separate of RB. Then there are wrinkles generated along the circumference and some elements have failed subsequently. Cracks appear in the thin section and extend along the circumference rapidly. The thin section is significantly damaged at $0.75 \mathrm{~ms}$ after the separate of $\mathrm{RB}$, and the bearing support has lost its load carrying capacity completely. It is considered that the current thickness of the thin section is the design target value for the current case.

\subsection{The width of the thin section}

Influence of axial width of the thin section to the failure of the section is discussed below. For the following cases, the thin section is set between the front flange and the maximum deformation position of the first buckling mode of the support, and its thickness is set at $60 \%$ of the thickness of the non-thinning section. To analyze the failure of the thin section with different width, we make the ratio of its axial width and wall thickness to be 10, 30 and 60 separately. The contour graphs of the effective plastic strain for different cases are shown in Fig 12.

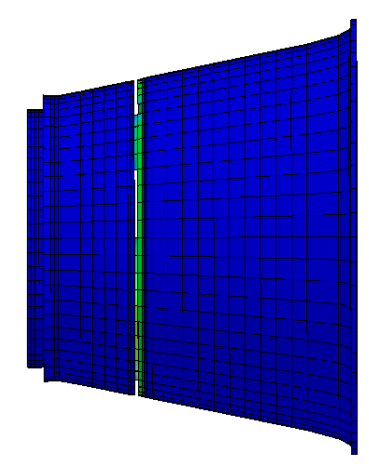

(a)The ratio is 10

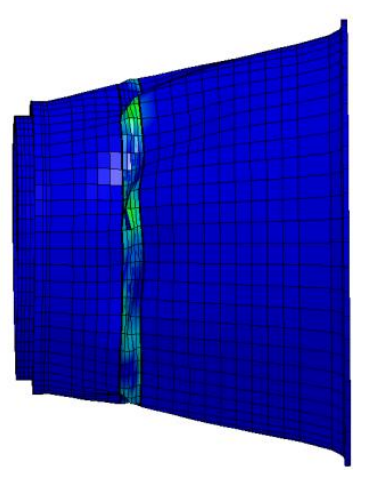

(b) The ratio is 30

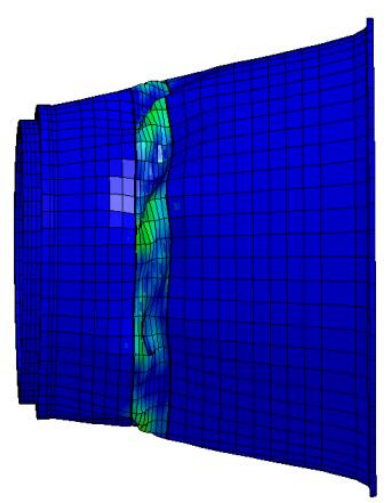

(c) The ratio is 60

Fig.12 Failure of the thin section with different width

When the ratio of axial width and wall thickness is 10 , the thin section fails without causing any wrinkles under the FBO load, as show in Fig.12 (a). However, effective plastic deformation in the form of wrinkling circumferentially around the thin section following the rotation of the FBO load are observed when the ratio of axial width and wall thickness of the thin section is 30 and 60 . This indicates that the ratio of the width and the thickness of the thin section will affect its failure mode under the FBO load. In order to achieve the energy absorption effect, it is necessary to ensure that the thin section can deform in the form of preferred wrinkling circumferentially. For the current case, this can be achieved when the ratio of axial width and wall thickness of the thin section is greater than 30 . 


\section{Summary}

A full-engine finite element model is utilized to analyze the influences of structural parameters of the thin section of the bearing support on failure mode after FBO event. Both implicit buckling mode analysis and explicit FBO simulation performed. The results show that the thin section will exhibit circumferential shear buckling failure when it is close to the front flange of the bearing support. And there is an axial position that the buckling coefficient of the bearing support is larger than other cases. The wall thickness of the thin section is directly related to the FBO peak load at bearing No.1. Effective plastic deformations in the form of wrinkling circumferentially around the thin section following the FBO load are observed when the ratio of axial width and wall thickness of the thin section is greater than 30 . The findings can be useful for the design of primary fuse structure.

\section{References}

[1]. Bird strikes to civil aircraft in China (2013) [D].Center of Aviation Safety Technology, CAAC, 2013. (in Chinese)

[2]. Sunil K.Sinha, Kevin E. Turner. Dynamic loading on turbofan due to bird-strike[C]. Proceeding of ASME Turbo Expo 2011. Canada.

[3]. FAR-33 Airworthiness standards: aircraft engines (Amend31) [M]. Washington, D.C, FAA, 2010.

[4]. CS-E Certification specifications for engines (Change3) [M]. EASA, 2010.

[5]. CCAR-33R2 China's civil aviation regulations of 33[M]. Beijing: Civil Aviation Administration of China, 2002(in Chinese).

[6]. Pablo Salas, Satchi Venkataraman. Optimum design of structural fuses for robust and predictable progressive failure [C]. 47th AIAA/ASME/ASCE/AHS/ASC Structures, Structural Dynamics, and Materials Conference. Newport, Rhode Island, 2006.

[7]. I.I. Ivanov, B.S. Blinnik. Dynamic loads acting on engine frame elements after fan blade out event study [C]. 29th Congress of the International Council of the Aeronautical Sciences. St. Petersburg, Russia, 2014.

[8]. John Andrew, Wahoo, Randy Marinus, et al. Bearing support fuse [P]. United States: US6447248B1, Sep.10, 2002.

[9]. Ernest Boratgis, James B. Coffin. Turbine engine bearing support [P]. US 6428269B1, Aug.8, 2002.

[10]. Thomas Matthew Doerflein, Stephen Anthony Wilton, Barry Lvnn Allmon. Method and apparatus for supporting rotor assemblies during unbalances[P]. United States: US6783319B2, Aug.31, 2004.

[11]. Luo G, Chen Wei, Zhao Zhenhua. Analysis Method of Critical Parameters on Aircraft Engines Bird Ingestion Airworthiness Certification[J]. Mechanical Science and Technology for Aerospace Engineering, 2016, 11(35): 1774 1779.

[12]. He Q, Xuan H.J, Liao L.F, et al. Numerical simulation of a thin plate impacted by blade projectile[J]. Engineering Mechanics, 2010, 27(4): 234-239.(in Chinese)

[13]. Liu L.L, Xuan H.J, Zhang N. Investigation on blade containment of aero-engine composite case[J]. Engineering Mechanics, 2013, 30(6): 314-319.(in Chinese)

[14]. Wen Y J.Parameterization and validity analysis on the response of flat blades due to bird impact [D]. Nanjing: Nanjing University of Aeronautics and Astronautics, 2006.(in Chinese)

[15]. Zou W S, Zuo Z X, Feng H H, et al. Application of the Sub-Model Method in the Engine Strength Analysis[J]. Journal of Beijing Institute of Technology, 2001, 10(3): 260 265. 